\title{
Integrating Text Chunking with Mixture Hidden Markov Models for Effective Biomedical Information Extraction
}

\author{
Min Song, Il-Yeol Song, Xiaohua Hu, and Robert B. Allen \\ College of Information Science \& Technology Drexel University, \\ Philadelphia, PA 19104 \\ (215) 895-2474, 01 \\ \{min.song, song, thu, rba\} adrexel.edu
}

\begin{abstract}
This paper presents a new information extraction (IE) technique, KXtractor, which integrates a text chunking technique with Mixture Hidden Markov Models (MiHMM). KXtractor is differentiated from other approaches in that (a) it overcomes the problem of the single Part-Of-Speech (POS) HMMs with modeling the rich representation of text where features overlap among state units such as word, line, sentence, and paragraph. By incorporating sentence structures into the learned models, KXtractor provides better extraction accuracy than the single POS HMMs do. (b) It resolves the issues with the traditional HMMs for IE that operate only on the semi-structured data such as HTML documents and other text sources in which language grammar does not play a pivotal role. We compared KXtractor with three IE techniques: 1) RAPIER, an inductive learning-based machine learning system, 2) a Dictionary-based extraction system, and 3) single POS HMM. Our experiments showed that KXtractor outperforms these three IE systems in extracting proteinprotein interactions. In our experiments, F-measure for KXtractor was higher than ones for RAPIER, a dictionary-based system, and single POS HMM respectively by $16.89 \%, 16.28 \%$, and $8.58 \%$. In addition, both precision and recall of KXtractor are higher than those systems.
\end{abstract}

\section{Introduction}

The proliferation of the biomedical literature available on the Web is overwhelming. While the amount of data available to us is constantly increasing, our ability to absorb and process this information remains a challenging task. The biomedical research domain has recently become a target domain that information extraction (IE) can be spotlighted on. IE is the process of scanning text for information relevant to some interest, including extracting entities, relations, and events. In this paper, we propose a novel IE technique, called KXtractor, Mixture Hidden Markov Models (MiHMMs) combined with a Support Vector Machine (SVM)-based text chunking technique.

MiHMM is defined as a mixture of Hidden Markov Models (HMMs) organized in a hierarchical structure to help the IE system cope with data sparseness. MiHMM takes a set of sentences with contextual cues that were identified by a Support Vector Machine-based text chunking technique. MiHMM then learns a generative 
probabilistic model of the underlying state transition structure of the sentence from a set of tagged training data. Given a trained probabilistic mixture model of the data, the system is able to apply this model to new unseen input documents to predict which portions of these sentences are likely targets according to the training data template. The paper investigates relationships between structure and performance of HMMs applied to information extraction problems.

KXtractor is different from existing HMMs as follows: (a) It employs probabilistic mixture of HMMs that are hierarchically structured. (b) It incorporates contextual and semantic cues into the learned models to extract knowledge from the unstructured text collections without any document structures. (c) It adopts an SVM text chunking technique to partition sentences into grammatically related groups. Thus, using KXtractor for extracting biomedical entities has the following advantages over other approaches: (a) it overcomes the problem of the single POS HMMs with modeling the rich representation of text where features overlap among state units such as word, line, sentence, and paragraph. By incorporating sentence structures into the learned models, KXtractor provides better extraction accuracy than the single POS HMM does. (b) it resolves the issues with the single POS HMMs for IE that operate only on the semi-structured data such as HTML documents and other text sources in which language grammar does not play a pivotal role.

With this novel and robust IE technique, we have extracted protein-protein pairs from abstracts in MEDLINE. We have compared the system performance of KXtractor with other IE techniques such as a rule-based learning, a dictionary-based, and single POS HMM techniques. Our experimental results show that KXtractor is superior to these techniques in most cases. The rest of the paper is organized as follows: Section 2 describes the overall architecture of KXtractor. Section 3 describes the evaluation. Section 4 reports on the experiments. Section 5 concludes the paper.

\section{The System Architecture}

Figure 1 illustrates the system architecture of KXtractor. The system consists of two major components: 1) sentence chunking by an SVM component and 2) relation extraction by MiHMM component.

In the sentence chunking by an SVM component, input data is plain text consisting of titles and abstracts. The input data is separated into sentences. A set of regular expression rules are applied to parse sentences. For a parsed sentence, we applied an integrated POS tagging technique proposed by Song et al. [9] to tag sentences with POS. With SVM-based text chunking technique, these POS tagged sentences are then grouped into chunks of different phrase types such as noun, verb, and preposition chunk. In the relation extraction by MiHMM component, MiHMM is applied to the grouped phrases by the SVM text chunking technique. The results of running KXtractor are a set of tuples related to protein-protein pairs. KXtractor stores these tuples in the knowledge base and resets the token statistics for the next input data. The detailed description of the components is provided in the sub-sections below. Figure 2 


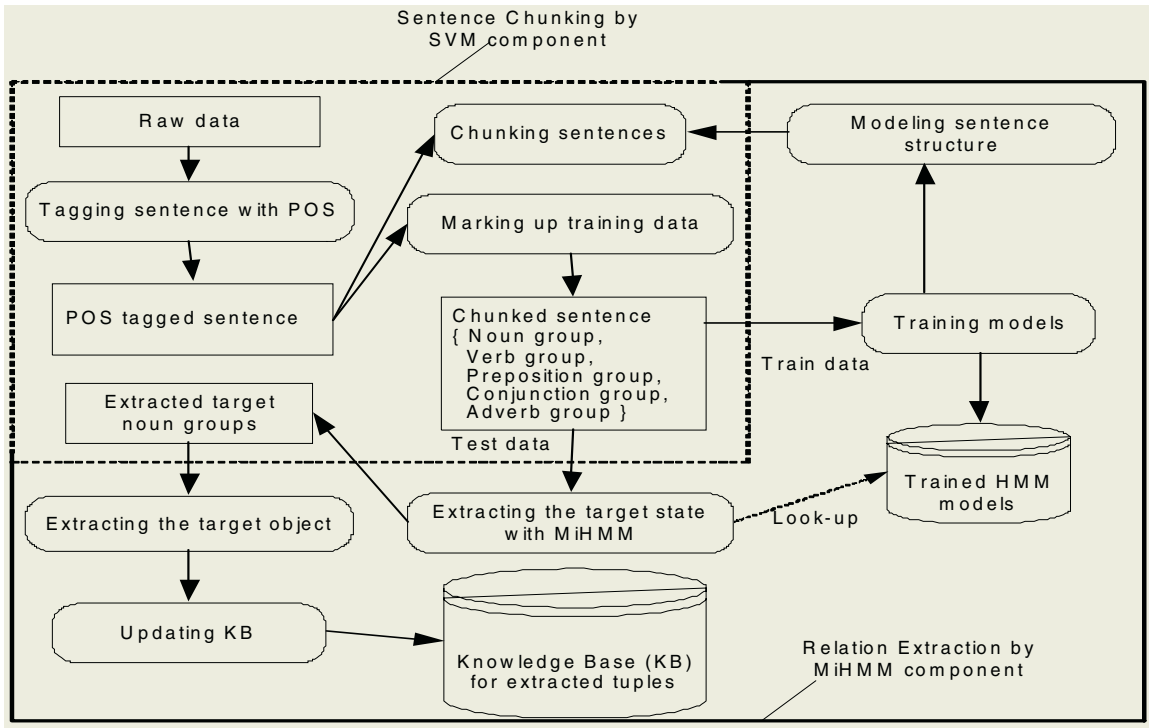

Fig. 1. System architecture of KXtractor

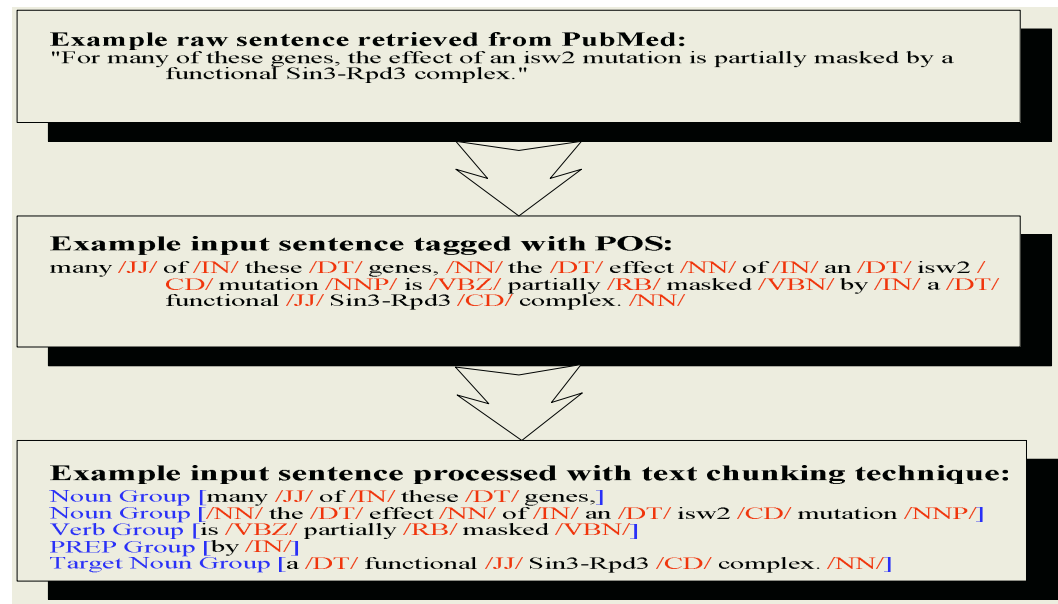

Fig. 2. A procedure of sentence parsing JJ denotes adjective, IN denotes preposition, DT denotes determiner, $\mathrm{CD}$ cardinal number, NN denotes singular noun, NNP denotes proper noun, VBZ denotes verb, VBN denotes verb, and RB denotes adverb

illustrates the procedure of converting a raw sentence from PubMed to the phrasebased units grouped by the SVM text chunking technique. The top box shows a sentence that is part of abstracts retrieved from PubMed. The middle box illustrates 
the parsed sentence by POS taggers. The bottom box shows the final conversion made to the POS tagged sentence by the SVM based text chunking technique.

\subsection{Sentence Chunking by an SVM Component}

Text chunking is defined as dividing a text in syntactically correlated parts of words [5]. Chunking is recognized as a series of the processes - first identifying proper chunks from a sequence of tokens (such as words), and second classifying these chunks into some grammatical classes. Major advantages of using text chunking over full parsing techniques are that partial parsing such as text chunking is much faster, more robust, yet sufficient for IE. Text chunking based on Support Vector Machines (SVMs) was reported to produce the highest accuracy in the text chunking task [5]. The SVMs-based approaches such as other inductive-learning approaches take as input a set of training examples (given as binary valued feature vectors) and find a classification function that maps them to a class. SVMs are known to robustly handle large feature sets and to develop models that maximize their generalizability. This makes them an ideal model for IE.

Generalizability in SVMs is based on statistical learning theory and on the observation that it is useful to misclassify some of the training data so that the margin between other training points is maximized [4]. This is particularly useful for real world data sets that often contain inseparable data points. Although training is generally slow, the resulting model is usually small and runs quickly. This is because only the patterns that help define the function that separates positive from negative examples. In addition, SVMs are binary classifiers and so we need to combine SVM models to obtain a multiclass classifier. Due to the nature of the SVM as a binary classifier it is necessary in a multi-class task to consider the strategy for combining several classifiers. In this paper, we use Tiny SVM [4] in that Tiny SVM performs well in handling a multi-class task.

\subsection{Relation Extraction by MiHMM Component}

Figure 3 is a schematic representation of how our MiHMM works. Our phrase group includes 14 phrase types. Our models are constructed with the assumption that the model is fully connected, which means that the model emits a segment of any type at any given position within the sentence. Bold boxes in Figure 3 indicate the target noun group that contains either proteins or a protein-protein pair. Each box represents a phrase group and the circles inside the box show the POS tags assigned to words in order, which appears in the sentence. The model is trained maximum likelihood parameter estimation. From the sentence training set we can easily obtain the information concerning the frequency that a given state or observation occurred and the frequency with which a state transition or observation emission was made. The parameters of the model are the transition probabilities $P\left(q \rightarrow q^{\prime}\right)$ that one state follows another and the emission probabilities $P\left(q \uparrow q^{\prime}\right)$ that a state emits a particular output symbol. The probability of a string $\mathrm{x}$ being emitted by an HMM is computed as a sum over all possible paths by: 


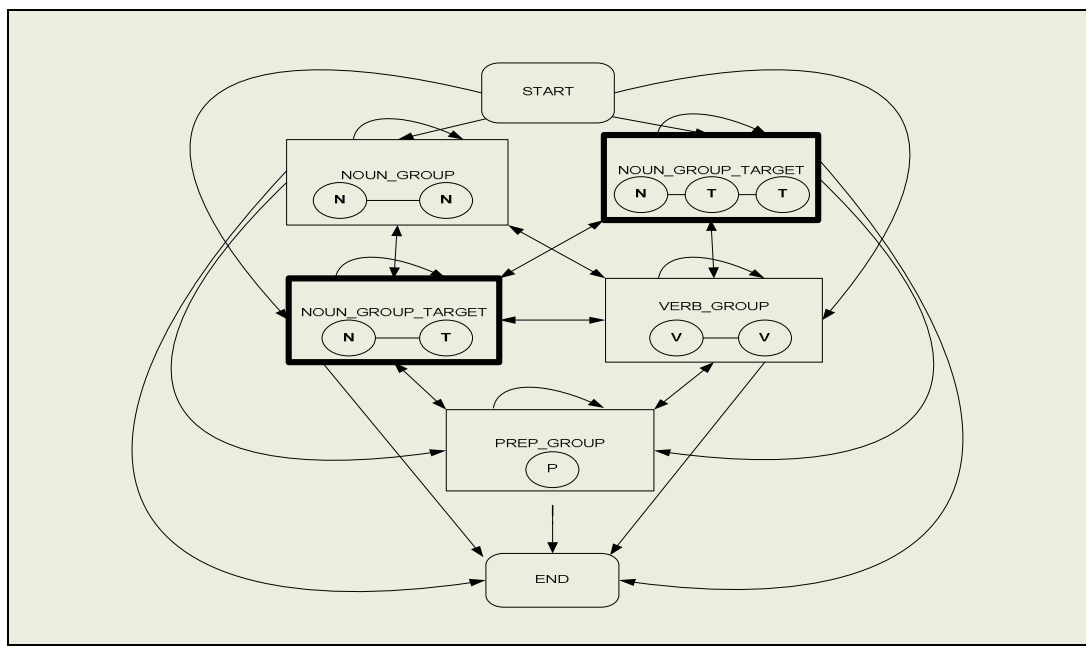

Fig. 3. Noun phrase based Mixture Hidden Markov ModelsN denote noun, $\mathrm{P}$ denotes preposition, $\mathrm{T}$ denotes target, and $\mathrm{V}$ denote verb

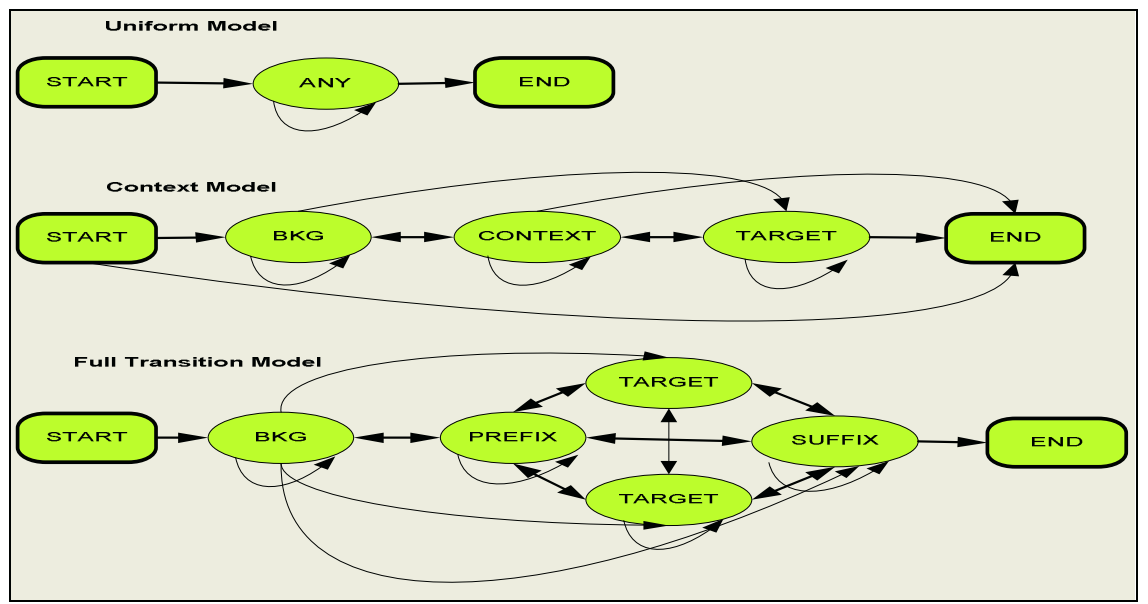

Fig. 4. Graphic representation of MiHMM. BKG denotes Background

$$
P(x \mid M)=\sum_{q_{1, \ldots}, q_{t} \in Q^{l}} \prod_{k=1}^{l+1} P\left(q_{k-1} \rightarrow q_{k}\right) P\left(q_{k} \uparrow x_{k}\right)
$$

where $q_{0}$ and $q_{l} 1$ are restricted to be $q_{I}$ and $q_{F}$ respectively, and is an end-ofstring token. The forward algorithm can be used to calculate this probability [7]. The observable output of the system is the sequence of symbols that the states emit, but the underlying start sequence itself is hidden. One common goal of learning problems 
that use HMMs is to recover the state sequence $V(x \mid M)$ that has the highest probability of having produced an observation sequence:

$$
V(x \mid M)=\underset{q_{1} \ldots q_{t} \in Q^{l}}{\arg } \prod_{k=1}^{l+1} P\left(q_{k-1} \rightarrow q_{k}\right) P\left(q_{k} \uparrow x_{k}\right)
$$

Determining this state sequence is efficiently performed by dynamic programming with the Viterbi algorithm [10].

\section{Evaluation}

To evaluate KXtractor, we compare it with three other well-known IE methods: 1) the dictionary-based extraction, 2) RAPIER, a rule-based machine learning extraction, and 3) single POS HMM. Performance of these IE systems is measured by precision, recall, and F-measure. The data used for experiments are retrieved from MEDLINE.

\subsection{Data Collection}

The IE task conducted in this paper is a multiple slot extraction task. The goal of our IE task is to extract instances of $n$-ary relations; that is, protein-protein interactions. The protein-protein interaction data sets are composed of abstracts gathered from the MEDLINE database [6]. MEDLINE contains bibliographic information and abstracts from more than 4000 biomedical journals. From this huge text corpus, we combined and utilized MEDLINE data sets provided by [8] and [2]. The data sets consist of 1700 MEDLINE records. These data sets characterize physical interactions between pairs of proteins. In terms of sentences, the data sets consist of 6417 positive and 46123 negative sentences. It contains 10123 instances of 913 protein-protein pairs. To label the sentences in these abstracts, we matched the target tuples to the words in the sentence. A sentence that contained words that matched a tuple was taken to be a positive instance. Every other sentence was considered to be a negative instance.

\subsection{Dictionary-Based Extraction}

We developed a Dictionary-based extraction system proposed by Blaschke et al. As described in [2], the following six steps were taken to extract protein-protein interactions: 1) the protein names are collected from the Database of Interacting Proteins (DIP) and Protein-Protein Interaction Database (PPID) databases. The synonyms of the target proteins are manually provided. 2) The 14 verbs, indicating actions related to protein interaction, are used. 3) Abstracts are provided from MEDLINE. 4) The passages containing target proteins and actions are identified. 5) The original text is parsed into fragments preceding grammatical separators. 6) The final step is to build protein-protein pairs.

\subsection{RAPIER}

To evaluate the performance of KXtractor, we compare KXtractor with RAPIER. RAPIER [3] is a well-known IE system that was developed with a bottom-up 
inductive learning technique for learning information extraction rules. In order to use the slot-filling IE systems like RAPIER for extracting relations, we adapt the Rolefiller approach proposed by Bunescu et al. [2]. The Role-filler approach allows for extracting the two related entities into different role-specific slots. For protein interactions, Bunescu et al. [2] name the roles interactor and interactee. As indicated by the role names, protein-protein interactions are defined with the assumption that proteins appear in the same sentence.

\subsection{Single POS HMM}

In order to verify that our MiHMM models are superior to the plain HMM, we develop a plain HMM, based on single terms and a single model that incorporate less grammatical information. We implemented single-level HMMs whose states emit words, but are typed with part-of-speech (POS) tags so that a give state can emit words with only a single POS. The Viterbi algorithm extracts information from documents modeled by an HMM. With the fix structure, the objective of learning is to give high probabilities to training documents. The result of learning is estimated probabilities for vocabularies and transitions.

\section{Experiments}

We conducted experiments to evaluate the performance of KXtractor on the task of protein-protein interaction extraction. In experiments the machine learning systems were trained using the abstracts with proteins and their interactions, processed by the text chunking technique. With these set of data, the IE systems extract interactions among these proteins. This gives us a measure of how the extraction systems for protein-protein pairs perform alone. Performance is evaluated using ten-fold cross validation and measuring recall and precision. As the task of interest is only to extract interacting protein-pairs, in our evaluation we do not consider matching exact position and every occurrence of interacting protein-pairs within the abstract.To evaluate our IE systems, we construct a precision-recall graph. Recall denotes the ratio of the number of slots the system found correctly to the number of slots in the answer key, and precision is the ratio of the number of correctly filled slots to the total number of slots the system filled.

Our experiments show that RAPIER produces relatively high precision but low recall. The similar results are observed in the dictionary-based extraction method which gives also high precision but low recall. Single POS HMM produces the second best results, although recall is relatively lower than precision. Among these three systems, KXtractor outperforms RAPIER, Dictionary, and single POS HMM in terms of precision, recall, and F-measure. As shown in Table 1, F-Measure of KXtractor is $52.38 \%$ whereas RAPER is $35.49 \%$, dictionary is $36.10 \%$, and single POS HMM is $43.80 \%$. Figure 5 shows the precision-recall graphs of KXtractor, RAPIER, Dictionary, and single POS HMM-based extraction for the protein-protein interaction data set. The curve for KXtractor is superior to the curves for RAPIER, Dictionary, and single POS HMM. 
Table 1. Comparison of extraction system performance

\begin{tabular}{|c|c|c|l|}
\hline Extraction System & Precision & Recall & F-Measure \\
\hline Dictionary-based extraction & $62.31 \%$ & $32.81 \%$ & $36.10 \%$ \\
\hline RAPIER & $60.17 \%$ & $34.12 \%$ & $35.49 \%$ \\
\hline Single POS HMM & $67.40 \%$ & $47.23 \%$ & $43.80 \%$ \\
\hline KXtractor & $70.23 \%$ & $51.21 \%$ & $52.38 \%$ \\
\hline
\end{tabular}

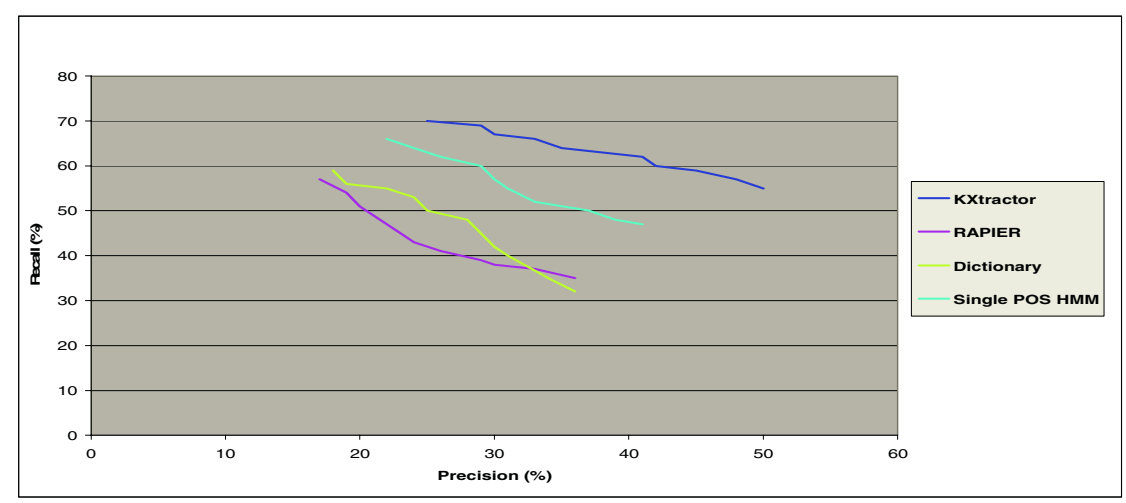

Fig. 5. Precision-recall graph for extracting protein-protein pairs

\section{Conclusion}

In this paper, we proposed a novel and high quality information extraction system, called KXtractor, a noun phrase-based Mixture Hidden Markov Models (MiHMM) system. KXtractor consists of two major components: 1) text chunking and 2) Mixture Hidden Markov Models (MiHMM) component. KXtractor is differentiated from other approaches in that (a) It overcomes the problem of the single POS HMMs with modeling the rich representation of text where features overlap among state units such as word, line, sentence, and paragraph. By incorporating sentence structures into the learned models, KXtractor provides better extraction accuracy than the single POS HMMs. (b) It resolves the issues with the single POS HMMs for IE that operate only on the semi-structured data such as HTML documents and other text sources in which language grammar does not play a pivotal role.

We compared KXtractor with three well-known IE techniques: 1) RAPIER, a rulebased machine learning system, 2) Dictionary-based extraction system which was proposed by [2], and 3) single POS HMM. Our experiments showed that KXtractor outperforms other IE techniques such as RAPIER, dictionary-based, and single POS HMM in extracting protein-protein interactions in terms of F-measure. The F- 
Measure of KXtractor is $52.38 \%$ whereas RAPER is $35.49 \%$, dictionary is $36.10 \%$, and single POS HMM is $43.80 \%$. In addition, both precision and recall of KXtractor are higher than those of RAPIER, Dictionary, and single POS HMM. In our follow-up paper, we will apply KXtractor to other types of relation extractions such as subcellular-localization relation extraction. We also plan to compare KXtractor with other IE systems such as MaxEnt and SVM.

\section{References}

1. Blaschke, C., Andrade, M.A., Ouzounis, C., and Valencia, A. (1999). Automatic Extraction of Biological Information from Scientific Text: Protein-Protein Interactions, In Proceedings of the Seventh International Conference on Intelligent Systems for Molecular Biology, Heidelberg, Germany, 60-67.

2. Bunescu, R., Ge, R., Kate, R.J., Marcotte, E.M., Mooney, R.J., Ramani, A.K., and Wong, Y.W. (2004). Comparative Experiments on Learning Information Extractors for Proteins and their Interactions. To appear in Journal Artificial Intelligence in Medicine (Special Issue on Summarization and Information Extraction from Medical Documents).

3. Califf, M.E. and Mooney, R.J. (1999). Relational Learning of Pattern-Match Rules for Information Extraction. Proceedings of the Sixteenth National Conference on Artificial Intelligence (AAAI-99), Orlando, FL, 328-334.

4. Cortes, C. and Vapnik, V. (1995). Support-Vector Networks. Machine Learning, 20(3): 273-297.

5. Kudo, T. and Matsumoto, Y. (2000). Use of Support Vector Learning for Chunk Identification. In Proceedings of CoNLL- 2000 and LLL-2000, Saarbruncken, Germany, 142-144.

6. National Library of Medicine (2003). The MEDLINE database, http://www.mcbi.nlm.nih.gov/PubMed/.

7. Rabiner, L. R. (1989). A Tutorial on Hidden Markov Models and selected applications in speech recognition. Proceedings of the IEEE, 77:257-286.

8. Skounakis, M., Craven, M., and Ray, S. (2003). Hierarchical Hidden Markov Models for Information Extraction. Proceedings of the 18th International Joint Conference on Artificial Intelligence, Acapulco, Mexico, August, 427-433.

9. Song, M, Song, I-Y., and Hu, X. (2003). KPSpotter: A Flexible Information Gain-based Keyphrase Extraction System, Fifth International Workshop on Web Information and Data Management (WIDM'03), New Orleans, Lousiana, 50-53.

10. Viterbi, A. J. (1967). Error bounds for convolutional codes and an asymptotically optimal decoding algorithm. IEEE Transactions on Information Processing, 13:260-269. 